\title{
Detection of a novel mutation in the ryanodine receptor gene in an Irish malignant hyperthermia pedigree: correlation of the IVCT response with the affected and unaffected haplotypes
}

Katherine E Keating, Linda Giblin, Patrick J Lynch, Kathleen A Quane, Mary Lehane, James J A Heffron, Tommie V McCarthy

\begin{abstract}
Defects in the ryanodine receptor (RYR1) gene are associated with malignant hyperthermia (MH), an autosomal dominant disorder of skeletal muscle and one of the main causes of death resulting from anaesthesia. Susceptibility to MH (MHS) is determined by the level of tension generated in an in vitro muscle contracture test (IVCT) in response to caffeine and halothane. To date, mutation screening of the RYR1 gene in MH families has led to the identification of eight mutations. We describe here the identification of a novel mutation, Arg552Trp, in the RYR1 gene, which is clearly linked to the MHS phenotype in a large, well characterised Irish pedigree. Considering that the RYR1 protein functions as a tetramer, correlation of the IVCT with the affected and unaffected haplotypes was performed on the pedigree to investigate if the normal RYR1 allele in affected subjects contributes to the variation in the IVCT. The results show that the normal RYR1 allele is unlikely to play a role in IVCT variation.

$(\Im$ Med Genet 1997;34:291-296)
\end{abstract}

Keywords: malignant hyperthermia; in vitro contracture test; ryanodine receptor; novel mutation.

Department of

Biochemistry,

University College, Lee

Maltings, Prospect

Row, Cork, Ireland

K E Keating

L Giblin

P J Lynch

K A Quane

J J A Heffron

T V McCarthy

Department of Anaesthesia, Cork University Hospital, Cork, Ireland

M Lehane

Correspondence to:

Dr McCarthy.

Received 24 May 1996 Revised version accepted for publication 7 November publicati 1996
Malignant hyperthermia (MH) is a potentially fatal, autosomal dominant disorder of skeletal muscle. ${ }^{12} \mathrm{MH}$ is triggered in susceptible people by volatile anaesthetics and depolarising muscle relaxants and is characterised by a pattern of physiological responses consistent with anaesthetic induced abnormalities in muscle excitation-contraction coupling. ${ }^{13}$ Fulminant MH typically presents with hypercontracture, metabolic and respiratory acidosis, tachycardia, raised temperatures, and death, if not treated with the postsynaptic muscle relaxant, dantrolene. ${ }^{12}$

Many susceptible patients present with some, but not all, of the classical signs with variable intensity when exposed to agents known to induce the condition. Some known susceptible patients have had previous triggering anaesthetics without any complications. The basis of the clinical variability is poorly understood, and the clinical diagnosis of $M H$ is often difficult. ${ }^{4}$ On account of this difficulty, Larach et $a \hat{P}$ proposed a six scale system for estimating the qualitative likelihood of $\mathrm{MH}$ in a given patient. Once a person is diagnosed as being susceptible to $\mathrm{MH}$, the anaesthetics which trigger this syndrome can be avoided. It has been estimated that about one third of subjects diagnosed as MHS will actually develop $\mathrm{MH}$ when administered with a $\mathrm{MH}$ triggering agent. ${ }^{6}$ Therefore, it seems that other genetic or external factors influence triggering of $\mathrm{MH}$ in susceptible subjects.

A standardised in vitro muscle contracture test (IVCT) has been established by the European Malignant Hyperthermia Group (EMHG) for investigation of MH susceptibility. ${ }^{7}$ The IVCT is based on the contracture tensions induced in muscle in vitro by caffeine and halothane and allows the following diagnosis to be made: $\mathrm{MH}$ susceptible (MHS), MH equivocal (MHE), and MH normal (MHN). A person is diagnosed as MHS if a threshold tension of $0.2 \mathrm{~g}$ or greater is generated at a caffeine concentration of $2 \mathrm{mmol} / 1$ and independently at a halothane concentration of $2 \%$. MHE denotes where the threshold tension is generated with caffeine (MHE(c)) but not halothane or vice versa $(M H E(h))$. The tensions generated in the IVCT in MH probands is variable. In a study of 40 subjects who have survived a clinical episode of $\mathrm{MH}, 35(88 \%)$ tested MHS and five tested MHE (12\%). ${ }^{8}$ The basis of the variability in the IVCT, like the variability seen in the clinical presentation of $\mathrm{MH}$, is not understood.

Earlier biochemical studies on porcine and human $M H$ indicated that the primary defect in MHS is in the skeletal muscle calcium release channel, commonly known as the ryanodine receptor (RYR1). ${ }^{910}$ This has been corroborated by the identification of mutations in the RYR1 gene which cosegregate with $M H$ in a significant portion of $\mathrm{MH}$ pedigrees. The RYR1 protein has a subunit size of about $560000 \mathrm{Da}$. This protein associates as a homotetramer forming an elaborate structure that acts both as a calcium release channel and a "foot" structure bridging the gap between the sarcoplasmic reticulum and the $t$ tubule in skeletal muscle. ${ }^{311}$ The RYR1 gene maps to human chromosome $19 \mathrm{q} 13.1^{12}$ and, to date, mutation screening of the RYR1 gene in MHS has led to the identification of eight mutations: 
Arg163Cys, ${ }^{13} \quad$ Gly248Arg, ${ }^{14}$ Gly341Arg, ${ }^{15}$ Ile403Met, ${ }^{13}$ Tyr522Ser, ${ }^{16}$ Arg614Cys, ${ }^{17}$ Gly2433Arg, ${ }^{18}{ }^{19}$ and Arg2434His, ${ }^{20}$ accounting for approximately $20 \%$ of cases of $\mathrm{MH}$. Four of these mutations, namely Arg163Cys, Ile403Met, Tyr522Ser, and Arg2434His, are also associated with central core disease (CCD), an inherited myopathy closely associated with MHS. The Arg614Cys mutation also occurs in swine and is responsible for all cases of porcine $\mathrm{MH}^{2{ }^{22}}$ Genetic heterogeneity has been reported in $\mathrm{MH} .{ }^{23}$ Alternative MHS loci have been proposed to reside on chromosome $17 \mathrm{q} 11.2-\mathrm{q} 24^{24}$ and chromosome $7 \mathrm{q} 11.23-\mathrm{q} 21.1 .^{25}$ In addition linkage has recently been established in a single large pedigree between MHS and markers on the q13.1 region of chromosome $3 .^{26}$

Considering that the RYR1 protein functions as a tetramer and that significant variability exists in the IVCT and in $\mathrm{MH}$ clinical expression, it is possible that the normal allele of the RYR1 gene might contribute to the variable expression of $\mathrm{MH}$ at the physiological and clinical level. In the work reported here we identify a new RYR1 mutation in a large well characterised Irish $\mathrm{MH}$ pedigree and we examine the correlation between the IVCT response with the affected and unaffected RYR1 haplotypes.

\section{Methods}

GENOMIC AND CDNA

Genomic DNA was extracted from peripheral blood as previously described. ${ }^{27}$ Total RNA was extracted from $100 \mathrm{mg}$ muscle biopsy samples by guanidine thiocyanate extraction. ${ }^{28}$ First, strand cDNA synthesis was performed using $200 \mathrm{ng}$ random hexamer primers, $1 \mu \mathrm{g}$ total RNA, and 15 U AVM Reverse Transcriptase in a $20 \mu \mathrm{l}$ reaction volume as described previously. ${ }^{13}$

PCR AMPLIFICATION

PCR amplification of RYR1 gene segments used overlapping primers designed from the published human RYR1 sequence. ${ }^{29}$ The RYR1 gene segment 1486-1755 bp was amplified with the forward primer 1486f: 5'TACACCACTGCTGCCCACTTT- 3 ' and the reverse primer 1755r: 5'-TGCTTGTCCAGGAGGGAGATG-3' using cDNA as template (annealing temperature $56^{\circ} \mathrm{C}$ ). Forward primer 1624f: 5'-AACTTGGACTGGCTGGTCAG-3' with primer 1755r was used in the amplification of genomic DNA (annealing temperature $54^{\circ} \mathrm{C}$ ). Amplified products were digested using the recommendations of the restriction enzyme manufacturer (New England Biolabs, USA). Genotyping of the single nucleotide polymorphisms C1079T, C1411T, C3456T, C5356T, C7872T, T8187C, T8586C, A9182G, G591A, A1668G, and $\mathrm{T} 2286 \mathrm{C}$ and the microsatellite markers; D19S190, RYR1 (Wis1), and D19S191 were as described previously. ${ }^{14} 2030-32$

SSCP ANALYSIS

PCR products for SSCP analysis were amplified with $\left[\alpha \mathrm{P}^{32}\right] \mathrm{dCTP}$ as label. SSCP analysis was performed as described previously. ${ }^{13}$ The samples were run on a $6 \%$ polyacrylamide gel (acrylamide: $\mathrm{N}, \mathrm{N}$-methylenebisacrylamide = 99:1) (1) at room temperature, (2) at room temperature with $5 \%$ glycerol, and (3) at $4{ }^{\circ} \mathrm{C}$ with $5 \%$ glycerol.

\section{CLONING AND SEQUENCING}

For sequencing of DNA, PCR products were end filled using the Klenow fragment and cloned into the SmaI site of the commercial vector pTZ18r (Pharmacia Biotech, Sweden). Dideoxy sequencing reactions were performed using universal forward and reverse primers. Eight clones of each sample were sequenced to distinguish actual base pair changes from changes that may be incorporated as a result of PCR artefacts.

\section{PATIENTS}

Subject 23 suffered a clinical episode of $\mathrm{MH}$ during anaesthesia (proband). Muscle samples were subsequently obtained from 20 people through the Cork MH testing centre. Although subject 5 was diagnosed as MHS, contracture data were unavailable. Subjects $5,6,8,15,16$, 17 , and 24 were tested for $\mathrm{MH}$ susceptibility before the establishment of the standardised European Malignant Hyperthermia Group protocol in 1984. All other subjects were investigated after 1984 using the standardised protocol.

\section{Results}

The CK1 family presented (fig 1) is one of the largest and best characterised Irish $\mathrm{MH}$ families ascertained to date. Of the 21 people tested for $\mathrm{MH}$ susceptibility within this pedigree, seven (subjects 5, 6, 8, 15, 16, 17, and 24) were tested before the establishment of the standarised European Malignant Hyperthermia Group protocol. The protocol used in Cork for testing the subjects before the standardisation of the IVCT in 1984 is essentially identical to the standardised protocol except that in some cases only one muscle strip from each person was tested and, in addition to the standard halothane and caffeine concentrations, tensions generated at one to three additional concentrations were also recorded for some of the strips. In previous mutation screening efforts, no RYR1 mutation was identified in this pedigree despite performing SSCP analysis of the entire RYR1 $15.5 \mathrm{~kb}$ cDNA under two different SSCP conditions. As MHS in this family was previously shown to be clearly linked to the RYR1 gene (a maximum lod score of 4.52 at $\theta=0.00$ in favour of linkage of MHS to the RYR1 locus was recorded) and as several of the MH RYR1 mutations cluster in the $\mathrm{N}$-terminal region of the RYR1 protein, the first $2 \mathrm{~kb}$ of the coding region of the RYR1 was re-examined under a further SSCP gel electrophoresis condition.

For SSCP analysis, cDNA was synthesised from total RNA isolated from skeletal muscle biopsy samples from subject 14 of the pedigree. In the primary screen none of the previously reported mutations was detected. However, in the total SSCP analysis, nine aberrant SSCP 


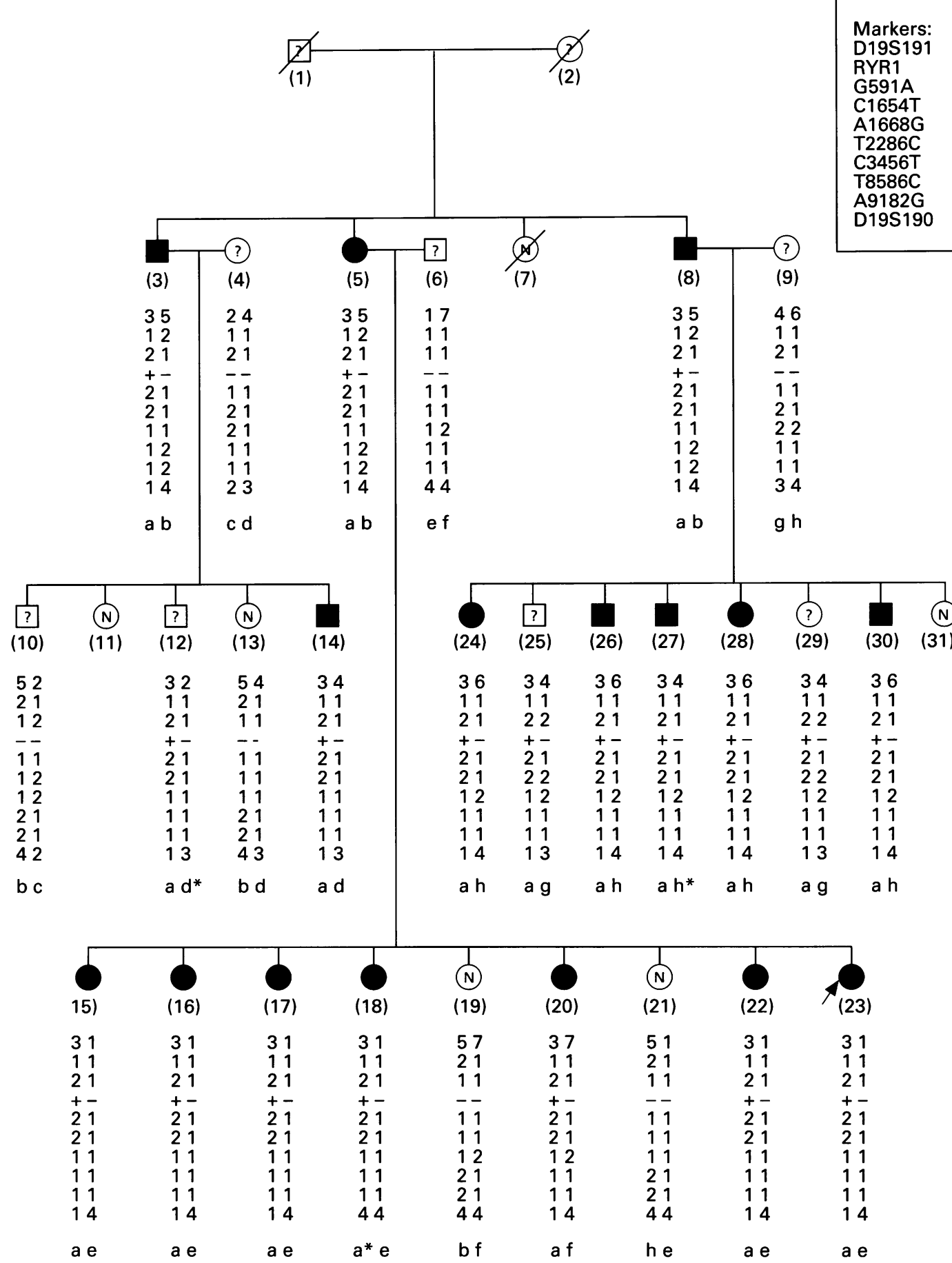

Figure 1 Pedigree of the CK1 MH family showing phenotypes and haplotypes. Pedigree symbols: shaded, MHS; clear with $N$ centred, MHN; clear with ? centred, status unknown. Haplotype analysis was performed with the markers listed. +/indicates the presence or absence of the C1654T mutation. The affected haplotype is labelled a, the predicted unaffected haplotypes are labelled $b$ to $h$ with the mutated allele assigned $a$. The asterisk denotes a recombinant haplotype. The arrow indicates the proband who suffered a $M H$ episode.

patterns were detected in different areas of the RYR1 gene from this subject. Further analysis of each PCR fragment with an aberrant SSCP showed that the polymorphisms G591A, C1079T, C1411T, T2286C, C5356T, C7872T, T8187C, and A9182G account for eight of the SSCP patterns. However, the unique SSCP pattern detected in the RYR1 segment 1486-1755 bp could not be accounted for by a known polymorphism. The amplified fragment of this RYR1 segment was blunt end cloned into the commercial vector pTZ18r. DNA sequencing showed a single substitution, $\mathrm{C} 1654 \mathrm{~T}$, and the previously reported polymor- phism A1668G. The C1654T substitution results in the replacement of an arginine with a tryptophan at position 552 in the RYR1 protein. The mutation results in the loss of a Sau3a restriction site allowing its detection by restriction enzyme cleavage.

In order to check for the presence of the C1654T missense mutation in the multiexon RYR1 gene in genomic DNA samples, it was necessary to design PCR primers that would allow amplification of the mutated region from genomic DNA. This was achieved by selecting PCR primers that closely flank the mutation. Using the primers $1624 \mathrm{f}(21 \mathrm{mer})$ and $1755 \mathrm{r}$ 


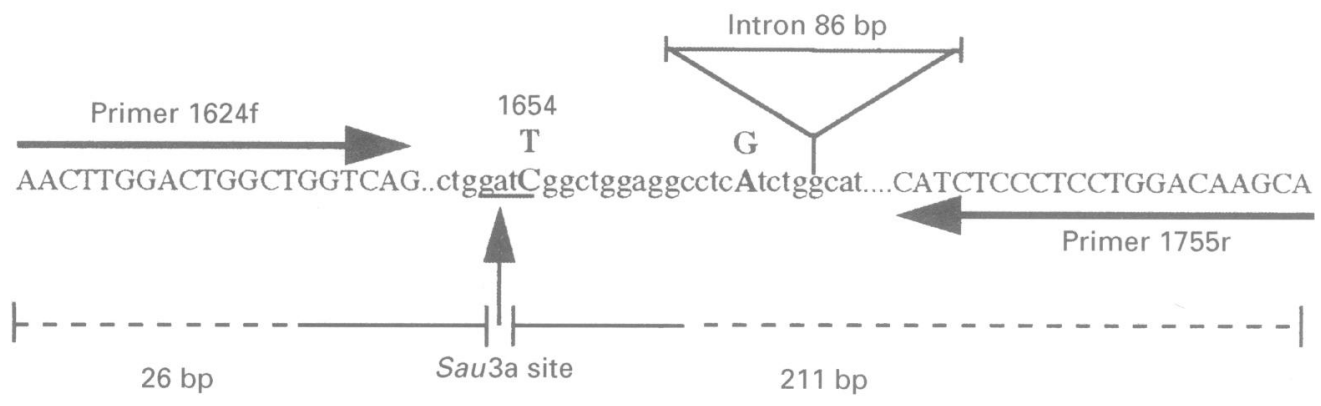

B
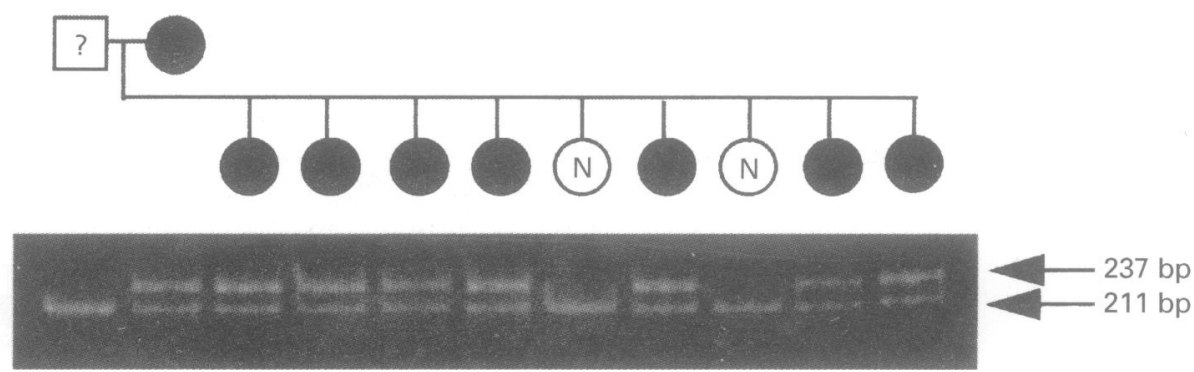

Figure 2 (A) A schematic representation of the RYR1 genomic fragment amplified with the genomic primers $1624 f$ and 1755r. The sequences of the forward primer $1624 \mathrm{f}$ and the reverse primer $1755 \mathrm{r}$ are in capital letters. Positions of the C1654T mutation and the polymorphism A1668G are in bold capital letters. The position of the $86 \mathrm{bp}$ intron is also indicated and the Sau3a restriction site underlined. (B) Sau3a restriction digest of the RYR1 genomic fragment amplified with primers $1624 \mathrm{f}$ and $1755 \mathrm{r}$ within a family unit of the pedigree. Pedigree symbols: shaded, MHS; clear with $N$ centred, $M H N$; clear with ? centred, status unknown. The C1654T mutation results in the loss of a Sau3a restriction site. Where a sample is homozygous normal, a restriction digest with Sau3 a of amplified DNA will produce restriction fragments of 211 $b p$ and $26 \mathrm{bp}$ in length. Where a sample is heterozygous for the mutation, the mutant allele will not be digested with the Sau 3 a enzyme resulting in an uncut fragment of $237 \mathrm{bp}$. The $26 \mathrm{bp}$ restriction fragment arising from the normal allele is not visible under the conditions used. The $237 \mathrm{bp}$ and $211 \mathrm{bp}$ products are indicated by arrows.

(21mer) it was possible to amplify a $237 \mathrm{bp}$ genomic fragment encompassing the C1654T mutation (fig $2 \mathrm{~A}$ ). The amplified fragment was $86 \mathrm{bp}$ larger than anticipated from the cDNA sequence, indicating the presence of an intron. Sequencing of the amplified 1624-1755 bp fragment of genomic DNA showed an intron at nucleotide position 1674 in the RYR1 gene (fig 2A).

To establish whether the C1654T missense mutation was present in the normal population, genomic DNA of 200 normal chromosomes were examined for the presence of the mutation by checking the appropriate amplified products for the loss of a Sau3a restriction site. None displayed the candidate mutation. The mutation was also absent in 97 additional unrelated MHS and four unrelated CCD patients indicating that this is a rare mutation. Sequence analysis showed that the mutation occurs on a RYR1 allele bearing the polymorphism A1668G. In order to determine if this polymorphism was associated with the C1654T mutation, the heterozygosity frequency of A1668G in 132 chromosomes was determined using SSCP and found to be 0.71 for allele A1668 and 0.29 for allele G1668. The $\mathrm{C} 1654 \mathrm{~T}$ mutation was not detected in any of these samples indicating that the C1654T mutation is a newly occurring mutation.

Segregation analysis of the C1654T mutation was performed in the family and showed that the mutation cosegregated precisely with the MHS phenotype in the family (figs 1 and
2B). Furthermore, conservation across species and RYR isoforms implicates $\operatorname{Arg} 552$ as a functionally important amino acid.

Affected and unaffected RYR1 haplotypes were constructed by genotyping the three RYR1 linked microsatellite markers D19S191, ${ }^{30}$ RYR1 (Wis1), ${ }^{31}$ D19S190, ${ }^{32}$ and the six RYR1 PCR-RFLPs G591A, A1668G, T2286C, C3456T, T8586C, and A9182G ${ }^{1420}$ in the pedigree (fig 1). The affected haplotype was labelled as a. Six unaffected haplotypes were discerned in subjects bearing the affected haplotype $a$ and labelled $b$ to $h$. Of the people diagnosed as MHS in the IVCT, subjects 24, $26,27,28$, and 30 shared the unaffected haplotype h while MHS subjects $15,16,17,18$, and 22 shared the unaffected haplotype e. MHS subjects 3 and 8 shared the unaffected $b$ haplotype while MHS subject 14 was the only person with the unaffected haplotype d (fig 1).

For each person, (1) the unaffected RYR1 haplotype, (2) the tension generated in each MHS subject at $2 \%$ halothane and $2 \mathrm{mmol} / \mathrm{l}$ caffeine, and (3) the minimal value of halothane and caffeine that generated a tension that equalled or exceeded the EMHG agreed threshold value of $0.2 \mathrm{~g}$ is presented in table 1 . The results show clearly that there is considerable variation in the IVCT responses generated by each MHS subject even though all of the affected subjects share the same Arg552Trp mutation. Since several affected subjects share the unaffected haplotype e or $h$, it was possible to compare the IVCT responses in affected 
Table 1 IVCT tension responses for MHS subjects from the CK1 MH family. The values given for each person are the average of the IVCT response of two muscle strips, except for subjects 8, 15, 17, and 24 where data from one muscle strip only were available

\begin{tabular}{|c|c|c|c|c|c|c|}
\hline \multirow[b]{2}{*}{ Subject } & \multirow[b]{2}{*}{ Unaffected RYR1 haplotype } & \multicolumn{2}{|c|}{ Threshold concentration } & \multicolumn{2}{|c|}{ Maximum contracture } & \multirow[b]{2}{*}{ Status } \\
\hline & & Halothane & Caffeine & $2 \%$ halothane & 2 mmolll caffeine & \\
\hline 3 & $\mathrm{~b}$ & 1.0 & 1.0 & 1.5 & 1.6 & MHS \\
\hline 8 & $\mathrm{~b}$ & 0.5 & 0.5 & 2.1 & 2.85 & MHS \\
\hline 14 & $\mathrm{~d}$ & 1.5 & 1.0 & 0.998 & 2.425 & MHS \\
\hline 15 & $\mathrm{e}$ & 0.5 & 1.0 & 2.85 & 1.25 & MHS \\
\hline 16 & e & 0.5 & 2.0 & 1.612 & 1.0 & MHS \\
\hline 17 & e & 0.5 & 2.0 & 5.125 & 0.2 & MHS \\
\hline 18 & e & 1.5 & 1.0 & 0.788 & 0.625 & MHS \\
\hline 20 & $\mathrm{f}$ & 1.0 & 0.5 & 1.325 & 1.507 & MHS \\
\hline 22 & $\mathrm{e}$ & 1.5 & 1.0 & 1.15 & 2.125 & MHS \\
\hline 24 & $\mathrm{~h}$ & 1.0 & 2.0 & 0.6 & 0.2 & MHS \\
\hline 26 & $\mathrm{~h}$ & 1.0 & 0.5 & 2.412 & 3.65 & MHS \\
\hline 27 & $\mathrm{~h}^{\star}$ & 1.0 & 0.5 & 1.825 & 1.813 & MHS \\
\hline 28 & $\mathrm{~h}$ & 1.0 & 0.5 & 2.688 & 2.84 & MHS \\
\hline 30 & $\mathrm{~h}$ & 1.0 & 1.5 & 0.970 & 0.525 & MHS \\
\hline
\end{tabular}

subjects sharing a common unaffected haplotype. The results show that there is considerable variation in response to the IVCT within each unaffected haplotype. At $2 \%$ halothane, the tension range extends from $0.788 \mathrm{~g}$ to $5.125 \mathrm{~g}$ for haplotype e and $0.6 \mathrm{~g}$ to $2.688 \mathrm{~g}$ for haplotype h. Similarly at $2 \mathrm{mmol} / \mathrm{l}$ caffeine the tension range extends from $0.2 \mathrm{~g}$ to $2.125 \mathrm{~g}$ for haplotype e and $0.2 \mathrm{~g}$ to $3.65 \mathrm{~g}$ for haplotype $\mathrm{h}$ (table 1).

Examination of the concentration of halothane required to reach the threshold value of 0.2 $g$ was $1 \%$ for haplotype $h$ and ranged from $0.5 \%$ to $1.5 \%$ for haplotype e. The concentration of caffeine required to reach the threshold value ranged from $1 \mathrm{mmol} / 1$ to $2 \mathrm{mmol} / 1$ for haplotype e and $0.5 \mathrm{mmol} / 1$ to $2 \mathrm{mmol} / 1$ for haplotype $h$. Thus despite the fact that all of the MHS subjects had the same mutation, significant variation was observed in the tensions generated in the IVCT. Furthermore, there was no correlation between the tensions generated with halothane versus those generated with caffeine. Indeed, subject 17 had one of the lowest responses to caffeine and one of the highest responses to halothane in the IVCT (table 1).

Interestingly, all of the affected subjects reached the threshold value of $0.2 \mathrm{~g}$ tension at or below $1.5 \%$ halothane and no MHE phenotype was observed in the family. This may indicate that the Arg552Trp mutation has high penetrance with respect to the IVCT phenotyping.

\section{Discussion}

In an effort to identify new mutations in the RYR1 gene causing $M H$, SSCP analysis was completed on the entire $15.5 \mathrm{~kb}$ RYR1 gene from a MHS affected member of a large, well characterised Irish $\mathrm{MH}$ pedigree. Sequencing of a unique aberrant SSCP showed the novel RYR1 mutation, C1654T, which segregated precisely with the MHS phenotype in the pedigree. This mutation is likely to have arisen from the deamination of a 5-methyl-C residue at the $\mathrm{CpG}$ sequence present at nucleotide position 1654 in the normal RYR1 gene. Analysis of the RYR1 gene from 97 unrelated MHS patients showed that this mutation was absent, indicating that the $\mathrm{C} 1654 \mathrm{~T}$ mutation is a rare mutation. The chances of the mutation being a coincidental polymorphism are remote as it is clearly linked to the MHS phenotype in the pedigree, has not been detected in 200 normal chromsomes tested, and is conserved across species and RYR1 isoforms.

The evidence to date indicates that $\mathrm{MH}$ and CCD mutations in the RYR1 gene make the calcium release channel more sensitive to activating concentrations of halothane, caffeine, and other stimulators of channel opening. ${ }^{33}{ }^{34}$ The molecular mechanism by which RYR1 mutations cause this hypersensitive gating is unknown. In mutant channels, enhanced rates of $\mathrm{Ca}^{2+}$ release from the sarcoplasmic reticulum during anaesthesia could result in the internal $\mathrm{Ca}^{2+}$ concentrations reaching a threshold level where triggering of $\mathrm{Ca}^{2+}$ induced $\mathrm{Ca}^{2+}$ release through the RYR1 channel occurred continuously. Such a mechanism would explain the sustained muscle contraction and the sustained glycolytic and aerobic metabolism seen in $\mathrm{MH}$. Mutations in the RYRl gene making the calcium release channel more sensitive to low concentrations of halothane and caffeine would also explain the physiological response observed in muscle samples from susceptible patients in the IVCT. The addition of caffeine or halothane (or ryanodine) to a MHS sample would lead to an increased amount of $\mathrm{Ca}^{2+}$ released through the channels, producing a corresponding increase in tension by comparison with a MHN sample.

The results presented show that there is considerable variation in the IVCT caffeine and halothane responses in the different affected subjects in the pedigree. The basis for variability within the IVCT is unclear. Large pedigrees such as the one presented here, which are well characterised at the IVCT and genetic level, offer an opportunity to identify factors contributing to the IVCT variability. Since the RYR1 calcium release channel functions as a homotetramer $^{3}$ and the IVCT tensions are linked to the levels of calcium released during the test, we postulated that the normal allele of the RYR1 gene might play a role in the variation in the IVCT response in MHS subjects within a pedigree. If the normal RYR1 allele is responsible for the variation one would expect that people sharing a particular normal allele and a given mutant allele might have a similar response. However, the analysis here 
showed that the normal RYR1 allele does not appear to play a role in IVCT variation, since the six subjects carrying the RYR1 mutant allele and the unaffected $h$ haplotype exhibited wide variation in their IVCT responses. Similarly, the five affected subjects bearing unaffected haplotype e also exhibited wide variation in their IVCT responses.

Understanding the basis of variation in the IVCT response is an important issue in $\mathrm{MH}$ diagnosis. The reason for this is that some apparently normal subjects exhibit a MHE reaction in the absence of a segregating MHS mutation, while some susceptible subjects carrying known mutations can exhibit a marginal MHE response in the IVCT. ${ }^{15}$ The MHE phenotype is poorly understood and until such time as the basis of $\mathrm{MHE}$ is understood, its existence diminishes the prospect of a genetic means for MHN diagnosis in $\mathrm{MH}$ pedigrees.

The work presented shows that the normal RYR1 allele is not likely to be a significant contributing factor to variation in the IVCT. However, even though a large, well characterised pedigree was used here, the total numbers investigated are small and it is possible that the unaffected RYR1 haplotype contributes to IVCT variation in pedigrees carrying other RYR1 mutations. Alternatively, other good candidates that might play a role in IVCT variation would be those proteins that interact with the RYR1 homotetramer, such as the FKBP12 protein $^{35}{ }^{36}$ and the dihydropyridine receptor. ${ }^{37}$ The sarcoplasmic reticulum $\mathrm{Ca}^{2+}$ ATPases which are responsible for the rapid uptake of $\mathrm{Ca}^{2+}$ from the myoplasm into the sarcoplasmic reticulum would also merit investigation. $\mathrm{Fi}$ nally, the contribution of experimental factors to the variation of the IVCT has not been assessed in detail and it remains to be determined what contribution such factors make to IVCT variation.

The first two authors made equal contributions to this Fund $(038165 / 1.5 / \mathrm{AH})$ and the Irish Health Research Board.

1 Ellis FR, Heffron JJA. Clinical and biochemical aspects of malignant hyperthermia. In: Atkinson R, Adams A, eds. Recent advances in anaesthesia and analgesia. Vol 15. New York: Churchill-Livingstone, 1985:173-207.

2 Britt BA. Dantrolene-an update. In: Britt BA, ed. Malignan hyperthermia. Amsterdam: Martinus-Nijhoff Press, 1987 325-67.

3 MacLennan DH, Phillips MS. Malignant hyperthermia. Science 1992;256:789-94.

4 Hackl W, Mauritz W, Schemper M, Winkler M, Sporn P, Steinbereithner K. Prediction of malignant hyperthermi Steinbereithner $\mathrm{K}$. Prediction of malignant hyperthermia susceptibility: statistical

5 Larach MG, Localio AR, Allen GC, et al. A clinical grading scale to predict malignant hyperthermia susceptibility. scale to predict malignant

6 Kalow W. Inheritance of malignant hyperthermia-a review of published data. In: Britt BA, ed. Malignant hyperthermia. Amsterdam: Martinus-Niihoff Press, 1987:155-79.

7 European Malignant Hyperthermia Group. A protocol for investigation of malignant hyperpyrexia susceptibility. $\mathrm{Br} f$ Anaesth 1984;56:1267-71

8 Ording $\mathrm{H}$. The European $\mathrm{MH}$ group: protocol for in vitro diagnosis of susceptibility to MH and preliminary results. In: Britt BA, ed. Malignant hyperthermia. Amsterdam: Martinus-Nijhoff Press, 1983:269-77.

9 Nelson TE. Abnormality in calcium release from skeleta sarcoplasmic reticulum of pigs susceptible to malignan hyperthermia. $\mathcal{F}$ Clin Invest 1983;72:862-70.

10 Lopez J, Alamo LA, Caputo C, Wilinski J, Ledezma D. Intracellular ionized calcium concentration in muscle from humans with malignant hyperthermia. Muscle Nerve 1985; 8:355-8.
11 Coronado R, Morrissette J, Sukhareva M, Vaughan DM. Structure and function of ryanodine receptors. $A m \mathcal{J}$ Physiol 1994;266:C1485-504.

12 MacKenzie AE, Korneluk RG, Zorzato F, et al. The human ryanodine receptor gene: its mapping to $19 \mathrm{q} 13.1$, placement in a chromosome 19 linkage group, and exclusion as the gene causing myotonic dystrophy. Am $\mathcal{f}$ Hum Genet the gene causing

13 Quane KA, Healy JMS, Keating KE, et al. Mutations in the ryanodine receptor gene in central core disease and malignant hyperthermia. Nat Genet 1993;5:51-5.

14 Gillard EF, Ostu K, Fujii J, et al. Polymorphisms and deduced amino acid substitutions in the coding sequence of the ryanodine receptor (RYR1) gene in individuals with malignant hyperthermia. Genomics 1992;13:1247-54.

15 Quane KA, Keating KE, Manning BM, et al. Detection of a novel common mutation in the ryanodine receptor gene in malignant hyperthermia: implications for diagnosis and heterogeneity studies. Hum Mol Genet 1994;3:471-6.

16 Quane KA, Keating KE, Healy JMS, et al. Mutation screening of the RYR1 gene in malignant hyperthermia: detection of a novel Tyr to Ser mutation in a pedigree with associated central cores. Genomics 1994;23:236-9.

17 Gillard EF, Ostu K, Fujii J, et al. A substitution of cysteine for arginine 614 in the ryanodine receptor is potentially causative of human malignant hyperthermia. Genomics 1991;11:751-5.

18 Keating KE, Quane KA, Manning BM, et al. Detection of a novel RYR1 mutation in four malignant hyperthermia pedigrees. Hum Mol Genet 1994;3:1855-8.

19 Philips MS, Khanna VK, DeLeon S, Frodis W, Britt BA MacLennan DH. The substitution of Arg for Gly 2433 in the human skeletal muscle ryanodine receptor is associated with malignant hyperthermia. Hum Mol Genet 1994;3: 2181-6.

20 Zhang Y, Chen HS, Khanna VK, et al. A mutation in the human ryanodine receptor gene associated with central core disease. Nat Genet 1993;5:46-9.

21 Fujii J, Ostu K, Zorzato F, et al. Identification of a mutation in the porcine ryanodine receptor that is associated with malignant hyperthermia. Science 1991;253:448-51.

22 Ostu K, Khanna VK, Archibald AL, MacLennan DH. Cosegregation of porcine malignant hyperthermia and a probable causal mutation in the skeletal muscle ryanodine receptor gene in backcross families. Genomics 1991;11:74450.

23 Levitt RC, Nouri N, Jedlicka AE, et al. Evidence for genetic heterogeneity in malignant hyperthermia susceptibility. Genomics 1991;11:543-7.

24 Levitt RC, Olckers A, Meyers S, et al. Evidence for the localization of a malignant hyperthermia susceptibility locus (MHS2) to human chromosome 17q. Genomics 1992;14:562-6.

25 Iles DE, Lehmann-Horn F, Scherer SW, et al. Localization of the gene encoding the $\alpha_{2} / \delta$-subunits of the L-type voltage-dependent calcium channel to chromosome $7 \mathrm{q}$ and analysis of the segregation of flanking markers in malignan hyperthermia susceptible families. Hum Mol Genet 1994;3 969-75.

26 Sudbrak R, Procaccio V, Klausnitzer M, et al. Mapping of a further malignant hyperthermia susceptibility locus to further malignant hyperthermia susceptibility locus to

27 Old JM, Higgs DR. In: Weatherall D, ed. Methods in haematology. Edinburgh: Churchill-Livingstone, 1983:74

28 Chomczynski P, Sacchi N. Single step method of RNA isolation by acid guanidinium thiocyanate-phenol-chloroform extraction. Anal Biochem 1987;162:156-9.

29 Zorzato F, Fujii J, Otsu K, et al. Molecular cloning of cDNA encoding human and rabbit forms of the $\mathrm{Ca}^{2+}$ release channel (ryanodine receptor) of skeletal muscle sarcoplasmic reticulum. $\mathcal{F}$ Biol Chem 1990;265:2244-56.

30 Iles DE, Segers B, deLong P, Alleman J, Wieringa B. Trinucleotide repeat polymorphism at the D19S190 locus. Nucleic Acids Res 1992;20:1170.

31 Couch FJ, Hogan K, McCarthy TV, Gregg RG. Dinucleotide repeat polymorphism at the RYR1 locus (19q13.1). Nucleic Acids Res 1991;19:5094.

32 Iles DE, Segers B, deLong P, Alleman J, Wieringa B. Dinucleotide repeat polymorphism at the D19S191 locus. Nucleic Acids Res 1992;20:1170.

33 Mickelson JR, Gallant EM, Litterer LA, Johnson KM Rempel WE, Louis CF. Abnormal sacroplasmic reticulum ryanodine receptor in malignant hyperthermia. $\mathcal{F} \mathrm{Biol} \mathrm{Chem}$ 1988;263:9310-15

34 Ostu K, Nishida K, Kimura Y, et al. The point mutation Arg615-Cys in the $\mathrm{Ca}^{2+}$ release channel of skeletal sarcoplasmic reticulum is responsible for hypersensitivity to caffeine and halothane in malignant hyperthermia. $\mathcal{f}$ Biol Chem 1994;269:9413-15.

35 Jayarman T, Brillantes AM, Timerman AP, et al. FK506 binding protein associated with the calcium release channel (ryanodine receptor). $\mathcal{F ~ B i o l ~ C h e m ~ 1 9 9 2 ; 2 6 7 : 9 4 7 4 - 7 . ~}$

36 Brillantes AB, Ondrias K, Scott A, et al. Stabilization of calcium release channel (ryanodine receptor) function by FK506-binding protein. Cell 1994;77:513-23

37 Marty I, Robert M, Villaz M, et al. Biochemical evidence fo a complex involving dihydropyridine receptor and ryanodine receptor in triad junctions of skeletal muscle. Proc Nat Acad Sci USA 1994;91:2270-4. 